\title{
Triangular form of Laurent polynomial matrices and their factorization
}

\author{
Kuchma M. I. ${ }^{1}$, Gatalevych A. I. ${ }^{2}$ \\ ${ }^{1}$ Lviv Polytechnic National University, \\ 12 S. Bandera Str., 79013, Lviv, Ukraine \\ ${ }^{2}$ Ivan Franko National University of Lviv, \\ 1 Universytetska Str., 79000, Lviv, Ukraine
}

(Received 1 August 2021; Revised 15 December 2021; Accepted 19 December 2021)

\begin{abstract}
The issue of the semiscalar equivalence of Laurent polynomial matrices is investigated and the triangular form of such matrices and their finite sets is established with respect to this equivalence. The theorem on regularization of a Laurent polynomial matrix is proved. This theorem is used in the problem of factorization of such matrices. The factorization criterion of a Laurent polynomial matrix with a regular multiplier with a predetermined Smith normal form is obtained.
\end{abstract}

Keywords: Laurent polynomial matrix, semiscalar equivalence, triangular form, Smith normal form, matrix factorization.

2010 MSC: 15A21, 15A23, 15A24

DOI: $10.23939 / \mathrm{mmc} 2022.01 .119$

\section{Introduction}

Polynomial matrix algebra is used in various areas of applied mathematics. In particular, it is a fundamental tool for investigating the dynamics of a linear system and for designing feedback control laws [1,2]. Efficient algorithms, based on elementary transformations of Laurent polynomial matrices and their factorizations, allow for a complete analysis of the system dynamics. Also in recent years they have also been used extensively in the areas of digital signal processing and communication. Examples of their applications include broadband adaptive sensor array processing, the description of multiple-input-multiple-output communication channels, broadband subspace decomposition, and also digital filter banks for subband coding or data compression [3,4]. This matrices are used to describe a convolutive mixing process, which occurs, for example, when a set of signals arrives at an array of sensors via multiple paths. The study of factorizations of the Laurent polynomial matrices is relevant and many problems in the areas of digital signal processing and communication [5], can be converted to algebraic problems over polynomial and Laurent polynomial rings, and can be solved using the existing of algebraic methods.

In [6] was introduced the notion of semiscalar equivalence of polynomial matrices of maximal rank over an algebraically closed field of characteristic zero (in particular, a field of complex numbers) and the lower triangular form of matrices established there played an important role in constructing of the matrix factorization theory. These results were later generalized for polynomial matrices over an arbitrary field $[7,8]$, and the so called standard form of matrix pairs with respect to generalized equivalence was established $[8,9]$. In [10] the conditions for the existence of symmetric equivalence for symmetric matrix polynomials over a ring with involution were investigated.

Note that similar form for one polynomial matrix over infinite field with the respect to right semiscalar equivalence of matrices was obtained in [11].

For simplicity, we agree to assume that the main field is a field of complex numbers.

Let $\mathbf{C}[x]$ be the ring of polynomials with complex coefficients: $p(x)=\sum_{i=0}^{m} p_{i} x^{i}$ with $m \in \mathbf{N}$, $p_{i} \in \mathbf{C}$. If $p_{m} \neq 0$, then the polynomial degree of $p(x)$ is $\operatorname{deg} p(x)=m$, if $p_{m}=1, p(x)$ is said to be monic. 
Let $\mathbf{C}\left[x, x^{-1}\right]$ be the ring of Laurent polynomial: $p(x)=\sum_{i=-l}^{m} p_{i} x^{i}$ with $l, m \in \mathbf{Z},-l \leqslant m, p_{i} \in \mathbf{C}$. Let us suppose that $p_{-l} p_{m} \neq 0$, then one can always factorize $p(x)=p_{m} x^{-l} q(x)$ with monic polynomial $q(x) \in \mathbf{C}[x]$. The degree of $p(x)$ is defined as the degree of $q(x)$ and is denoted as: $\operatorname{deg} p(x)=m+l$. According to these considerations, $\mathbf{C}\left[x, x^{-1}\right]$ is an Euclidean ring, so a principal ideal domain. Hence $\mathbf{C}\left[x, x^{-1}\right]$ is an elementary divisor domain [12]. The invertible elements of $\mathbf{C}\left[x, x^{-1}\right]$ are non-zero monomials: $p(x)=a x^{\alpha}$ with $a \in \mathbf{C}^{*}=\mathbf{C} \backslash\{0\}$ and $\alpha \in \mathbf{Z}$. As greatest common divisors (g.c.d.) are defined up to an invertible element, one can set for uniqueness purposes, the g.c.d. to be a monic polynomial of $\mathbf{C}[x]$.

Let $M_{n}(\mathbf{C}[x])$ and $M_{n}\left(\mathbf{C}\left[x, x^{-1}\right]\right)$ be a ring of polynomial $n \times n$ matrices and a ring of polynomial Laurent $n \times n$ matrices (a ring of quasipolynomials), respectively, $G L_{n}(\mathbf{C}[x])$ and $G L_{n}\left(\mathbf{C}\left[x, x^{-1}\right]\right)$ their corresponding general linear groups.

By upper (lower) degree of Laurent polynomial matrix $A(x)$ of the form $A(x)=A_{-l} x^{-l}+\ldots+$ $A_{0}+\ldots+A_{m} x^{m}, A_{i} \in M_{n}(\mathbf{C})$, we will understand the number $m=\overline{\operatorname{deg}} A(x)$ with matrix coefficient $A_{m} \neq \mathbf{0},\left(-l=\underline{\operatorname{deg}} A(x)=-\overline{\operatorname{deg}} A\left(x^{-1}\right)\right.$ with matrix coefficient $\left.A_{-l} \neq \mathbf{0}\right)$ (where $\mathbf{0}$ denotes the $n \times n$ zero matrix). The degree of Laurent polynomial matrix $A(x)$ is defined as:

i.e. $s=m+l$.

$$
\operatorname{deg} A(x)=\overline{\operatorname{deg}} A(x)-\underline{\operatorname{deg}} A(x),
$$

Definition 1. Laurent polynomial matrix $A(x)=\sum_{i=-l}^{m} A_{i} x^{i}, A_{i} \in M_{n}(\mathbf{C})$ is called a regular if $\operatorname{det} A_{-l} \neq 0, \operatorname{det} A_{m} \neq 0$. If leading matrix coefficient $A_{m}=E$ (where $E$ denotes the $n \times n$ identity matrix), then the quasipolynomial matrix $A(x)$ is called a monic.

The aim of this article is to investigate the problem of semiscalar equivalence for Laurent polynomial matrices, to establish a triangular form of such matrices and their finite sets with respect to this equivalence. This can be done given that the ring of quasipolynomial matrices is an extension of the ring of polynomial matrices. Also derive the regularization condition for Laurent polynomial matrices and use it in the study of the factorization problem of Laurent polynomial matrices, i.e. the isolation of a regular factor with a predetermined Smith form from a nonsingular quasipolynomial matrix.

Due to the introduced notion of the value of the matrix on the system of roots of diagonal elements in [12] the process of establishing the conditions of regularization of the matrix polynomial and factorization of symmetric matrix polynomials is significantly simplified [13].

Definition 2. Laurent polynomial matrices $A(x)$ and $B(x)$ in the ring $M_{n}\left(\mathbf{C}\left[x, x^{-1}\right]\right)$ is called semiscalar equivalent if there exist such matrices $Q \in G L_{n}(\mathbf{C})$ and $S(x) \in G L_{n}\left(\mathbf{C}\left[x, x^{-1}\right]\right)$ that

$$
A(x)=Q B(x) S(x) \text {. }
$$

Definition 3. Laurent polynomial matrices $A(x)$ and $B(x)$ in the ring $M_{n}\left(\mathbf{C}\left[x, x^{-1}\right]\right)$ is called scalar equivalent if there exist such matrices $S, T \in G L_{n}(\mathbf{C})$ that

$$
A(x)=S B(x) T .
$$

Denote by $S_{A}(x)$ the Smith normal form of Laurent polynomial matrix $A(x)$ :

$$
S_{A}(x)=P(x) A(x) Q(x)=\operatorname{diag}\left(\varepsilon_{1}(x), \varepsilon_{2}(x), \ldots, \varepsilon_{n}(x)\right),
$$

where $P(x), Q(x) \in G L_{n}\left(\mathbf{C}\left[x, x^{-1}\right]\right), \varepsilon_{i}(x)$ are the invariant quasipolynomials, $\varepsilon_{i}(x) \mid \varepsilon_{i+1}(x), i=$ $1, \ldots, n-1$.

\section{Semiscalar equivalence of Laurent polynomial matrices}

In this section we will show that by semiscalar transformations each Laurent polynomial matrix can be reduced to a triangular form with invariant factors on the main diagonal. We will generalize this result for finite sets of quasipolynomial matrices and we will show that these sets of matrices have triangular forms, which are obtained using the same left and different right transformation matrices. 
Theorem 1. Let $A(x)$ be a nonsingular Laurent polynomial matrix over $\mathbf{C}\left[x, x^{-1}\right]$ and $\operatorname{rank} A(x)=$ $n$. Then for matrix $A(x)$ there exist such matrices $C \in G L_{n}(\mathbf{C})$ and $R(x) \in G L_{n}\left(\mathbf{C}\left[x, x^{-1}\right]\right)$ that

$$
C A(x) R(x)=\left\|\begin{array}{cccc}
\varepsilon_{1}(x) & 0 & \cdots & 0 \\
a_{21}(x) & \varepsilon_{2}(x) & & 0 \\
\vdots & \vdots & \ddots & \vdots \\
a_{n 1}(x) & a_{n 2}(x) & \ldots & \varepsilon_{n}(x)
\end{array}\right\|
$$

where the invariant factors $\varepsilon_{j}(x) \mid a_{i j}(x), i>j$ and they are the same as in the relation (1).

Proof. Let us consider the matrix $A_{1}(x)=A(x) x^{l}$ where $l=\underline{\operatorname{deg}} A(x)$. This matrix is a polynomial matrix. Using the notion of semiscalar equivalence for polynomial matrices [6], we obtain that there exist matrices $C \in G L_{n}(\mathbf{C})$ and $R_{1}(x) \in G L_{n}(\mathbf{C}[x])$ that

$$
C A_{1}(x) R_{1}(x)=\left\|\begin{array}{cccc}
\varepsilon_{1}^{\prime}(x) & 0 & \cdots & 0 \\
b_{21}(x) & \varepsilon_{2}^{\prime}(x) & & 0 \\
\vdots & \vdots & \ddots & \vdots \\
b_{n 1}(x) & b_{n 2}(x) & \ldots & \varepsilon_{n}^{\prime}(x)
\end{array}\right\|
$$

where $\varepsilon_{i}^{\prime}(x), i=1, \ldots, n$, are the invariant polynomials of the matrix $A_{1}(x)$ and $\varepsilon_{i}^{\prime}(x)$ divides all the elements of the column to which it belongs $\varepsilon_{j}^{\prime}(x) \mid b_{i j}(x), i>j$ and $\varepsilon_{i}^{\prime}(x)=x^{l_{i}} \varepsilon_{i}(x), \sum_{i=1}^{n} l_{i}=l, \varepsilon_{i}(x)$, $i=1, \ldots, n$ are the invariant quasipolynomials of $A(x)$. Since the elements of the form $x^{l_{i}}$ are invertible in the ring $\mathbf{C}\left[x, x^{-1}\right]$, then by the right elementary transformations we can reduce the matrix (3) to the form

$$
C A(x) x^{l} R_{1}(x)=\left\|\begin{array}{cccc}
\varepsilon_{1}(x) & 0 & \cdots & 0 \\
a_{21}(x) & \varepsilon_{2}(x) & & 0 \\
\vdots & \vdots & \ddots & \vdots \\
a_{n 1}(x) & a_{n 2}(x) & \ldots & \varepsilon_{n}(x)
\end{array}\right\| T(x)
$$

where $T(x)=\operatorname{diag}\left(x^{l_{1}}, x^{l_{2}}, \ldots, x^{l_{n}}\right)$ is invertible matrix in $M_{n}\left(\mathbf{C}\left[x, x^{-1}\right]\right)$. Right-multiplying (4) by the matrix $T(x)^{-1}$, we obtain equality (2) where

$$
x^{l} R_{1}(x) T(x)^{-1}=R(x) \in G L_{n}\left(\mathbf{C}\left[x, x^{-1}\right]\right) .
$$

The theorem is proved.

Remark 1. The lower triangular form of the matrix $A(x)$ from the equation (2) will hereinafter be called the triangular form or the standard form of Laurent polynomial matrix.

Theorem 1 can be generalized to the case of a finite set of Laurent polynomial matrices.

Theorem 2. Let $A_{1}(x), A_{2}(x), \ldots, A_{k}(x) \in M_{n}\left(\mathbf{C}\left[x, x^{-1}\right]\right)$ be a nonsingular Laurent polynomial matrices and $\operatorname{rank} A_{j}(x)=n, j=1, \ldots, k$. Then there exist such matrices $C \in G L_{n}(\mathbf{C}), R_{j}(x) \in$ $G L_{n}\left(\mathbf{C}\left[x, x^{-1}\right]\right), j=1, \ldots, k$ that

$$
C A_{j}(x) R_{j}(x)=\left\|\begin{array}{cccc}
\varepsilon_{1}^{(j)}(x) & & & 0 \\
& \varepsilon_{2}^{(j)}(x) & & \\
& & \ddots & \\
* & & & \varepsilon_{n}^{(j)}(x)
\end{array}\right\|, \quad j=1, \ldots, k
$$

where $\varepsilon_{i}^{(j)}(x)$ are the invariant factors of quasipolynomial matrices $A_{j}(x), i=1, \ldots, n, j=1, \ldots, k$. 
Proof. Let $A_{1}(x), A_{2}(x), \ldots, A_{k}(x)$ be a nonsingular Laurent polynomial matrices and

$$
s=-\min _{j=1}^{k}\left\{\underline{\operatorname{deg}} A_{j}(x)\right\} .
$$

Matrices $B_{j}(x)=A_{j}(x) x^{s}, j=1, \ldots, k$ are the polynomial matrices. According to [6,7] there exist such matrices $C \in G L_{n}(\mathbf{C}), R_{j}(x) \in G L_{n}\left(\mathbf{C}\left[x, x^{-1}\right]\right), j=1, \ldots, k$ that

$$
C B_{j}(x) R_{j}^{\prime}(x)=\left\|\begin{array}{cccc}
\varepsilon_{1}^{\prime(j)}(x) & & & 0 \\
& \varepsilon_{2}^{\prime(j)}(x) & & \\
& & \ddots & \\
* & & & \varepsilon_{n}^{\prime(j)}(x)
\end{array}\right\|, \quad j=1, \ldots, k
$$

where $\varepsilon_{i}^{\prime(j)}(x)=x^{s_{i j}} \varepsilon_{i}^{(j)}(x), \sum_{i=1}^{n} s_{i j}=s, j=1, \ldots, k, \varepsilon_{i}^{\prime(j)}(x)$ are invariant polynomials of $B_{j}(x)$ and $\varepsilon_{i}^{(j)}(x)$ are invariant quasipolynomials of $A_{j}(x), i=1, \ldots, n, j=1, \ldots, k$.

By the right elementary transformations we can reduce the matrix (6) to the form

$$
C A_{j}(x) x^{s} R_{j}^{\prime}(x)=\left\|\begin{array}{cccc}
\varepsilon_{1}^{(j)}(x) & & & 0 \\
& \varepsilon_{2}^{(j)}(x) & & \\
& & \ddots & \\
* & & & \varepsilon_{n}^{(j)}(x)
\end{array}\right\| T_{j}(x), \quad j=1, \ldots, k
$$

where $T_{j}(x)=\operatorname{diag}\left(x^{s_{1 j}}, x^{s_{2 j}}, \ldots, x^{s_{n j}}\right), j=1, \ldots, k$ are invertible matrices in $M_{n}\left(\mathbf{C}\left[x, x^{-1}\right]\right)$. Right-multiplying $j$-th relation by the matrix $T_{j}(x)^{-1}, j=1, \ldots, k$ we obtain equality (5), where $x^{s} R_{j}^{\prime}(x) T_{j}(x)^{-1}=R_{j}(x), j=1, \ldots, k$ are invertible matrices in $M_{n}\left(\mathbf{C}\left[x, x^{-1}\right]\right)$.

The theorem is proved.

Definition 4. It is said that Laurent polynomial matrix $A(x)=\sum_{i=-l}^{p} A_{i} x^{i}$ right regularized if there exists such matrix $R(x) \in G L_{n}\left(\mathbf{C}\left[x, x^{-1}\right]\right)$ that

$$
A(x) R(x)=A_{-s_{1}} x^{-s_{1}}+\ldots+A_{0}+\ldots+A_{s_{2}} x^{s_{2}}
$$

is regular matrix, i.e. $\operatorname{det} A_{-s_{1}} \neq 0, \operatorname{det} A_{s_{2}} \neq 0$.

The following theorem establishes a connection between scalar and semiscalar equivalences of Laurent polynomial matrices.

Theorem 3. Let

$$
A(x)=\sum_{i=-l}^{p} A_{i} x^{i}, \quad A_{i} \in M_{n}(\mathbf{C})
$$

and

$$
B(x)=\sum_{i=-l}^{p} B_{i} x^{i}, \quad B_{i} \in M_{n}(\mathbf{C})
$$

be are regular Laurent polynomial matrices with the triangular forms (2), respectively,

$$
T_{A}(x)=L A(x) V(x) \quad \text { and } \quad T_{B}(x)=K B(x) W(x)
$$

with $L, K \in G L_{n}(\mathbf{C}), V(x), W(x) \in G L_{n}\left(\mathbf{C}\left[x, x^{-1}\right]\right)$.

Quasipolynomial matrices $A(x)$ and $B(x)$ are scalar equivalent if and only if their triangular forms $T_{A}(x)$ and $T_{B}(x)$ are semiscalar equivalent. 
Proof. Necessity. Let us suppose that $A(x)$ and $B(x)$ are scalar equivalent, i.e. $A(x)=S B(x) T$ with $S, T \in G L_{n}(\mathbf{C})$.

By (7) we obtain

$$
T_{A}(x)=L A(x) V(x)=L S B(x) T V(x)=L S K^{-1} T_{B}(x) W(x)^{-1} T V(x) .
$$

Denote $C=L S K^{-1}$ and $Q(x)=W(x)^{-1} T V(x)$. We see that these matrices are invertible over $\mathbf{C}$ and $\mathbf{C}\left[x, x^{-1}\right]$ respectively. By (8) it follows that

$$
T_{A}(x)=C T_{B}(x) Q(x) .
$$

Thus, the triangular forms $T_{A}(x)$ and $T_{B}(x)$ respectively of the matrices $A(x)$ and $B(x)$ are semiscalar equivalent.

Proof of sufficiency directly follows from the fact that regular semiscalar equivalent matrices are scalar equivalent.

The theorem is proved.

\section{Theorem on the regularization of Laurent polynomial matrices}

In this section we will prove the theorem on the regularization of Laurent polynomial matrices in terms of value of the matrix on a system of roots of diagonal elements of matrix $[12,14]$ and we apply it to the problem of isolation a regular multiplier with a predetermined Smith form from the quasipolynomial matrix.

Definition 5 (see [14]). The matrix of the form

$$
M_{G(x)}(\Phi)=\left\|\begin{array}{c}
M_{g_{1}(x)}\left(\varphi_{1}\right) \\
M_{g_{2}(x)}\left(\varphi_{2}\right) \\
\ldots \\
M_{g_{n}(x)}\left(\varphi_{n}\right)
\end{array}\right\|
$$

is called value of the matrix $G(x)$ on a system of roots of diagonal elements of matrix $\Phi(x)=$ $\operatorname{diag}\left(\varphi_{1}(x), \varphi_{2}(x), \ldots, \varphi_{n}(x)\right)$, where $M_{g_{i}(x)}\left(\varphi_{i}\right)$ is the value of polynomial matrix on a system of roots of polynomial

$$
\varphi_{i}(x)=\left(x-\alpha_{1}\right)^{s_{1}}\left(x-\alpha_{2}\right)^{s_{2}} \cdot \ldots \cdot\left(x-\alpha_{m}\right)^{s_{m}},
$$

introduced in [12] as follows:

$$
M_{g_{i}(x)}\left(\varphi_{i}\right)=\left\|\begin{array}{c}
H_{1} \\
H_{2} \\
\ldots \\
H_{m}
\end{array}\right\|, \quad H_{k}=\left\|\begin{array}{c}
g_{i}\left(\alpha_{k}\right) \\
g_{i}^{\prime}\left(\alpha_{k}\right) \\
\ldots \\
g_{i}^{\left(s_{k}-1\right)}\left(\alpha_{k}\right)
\end{array}\right\|,
$$

where $g_{i}^{(j)}(x)$ are the derivatives of the $j$-th order of the matrix $g_{i}(x)$.

This definition is true in the case of quasipolynomial matrices $G(x)$ and $\Phi(x)$, given that the elements of the form $x^{l}$ are invertible in the ring $\mathbf{C}\left[x, x^{-1}\right]$.

Definition 6 (see [15]). Diagonal matrix

$$
\Phi(x)=\operatorname{diag}\left(\varphi_{1}(x), \varphi_{2}(x), \ldots, \varphi_{n}(x)\right)
$$

is called $d$-matrix if $\varphi_{i}(x) \mid \varphi_{i+1}(x), i=1, \ldots, n-1$.

Suppose that Laurent polynomial matrix $A(x)$ has the Smith form $S_{A}(x)$. 
Theorem 4. Let $A(x)$ be a Laurent polynomial matrix. Then there exists a matrix $R(x) \in$ $G L_{n}\left(\mathbf{C}\left[x, x^{-1}\right]\right)$ such that $A(x) R(x)$ is a regular quasipolynomial matrix of degree $s$ if and only if

$$
\begin{aligned}
& \text { 1) } \operatorname{deg} \operatorname{det} S_{A}(x)=n s, \\
& \text { 2) } \operatorname{det} M_{P(x)\left\|E x^{-s+1}, \ldots, E x^{-1}, E\right\|}\left(S_{A}\right) \neq 0,
\end{aligned}
$$

where $P(x) \in G L_{n}\left(\mathbf{C}\left[x, x^{-1}\right]\right)$ is the matrix of relation (1).

Proof. Necessity. Let us suppose that matrix $A(x)$ can be right regularized, i.e.

$$
A(x)=\left(A_{-s_{1}} x^{-s_{1}}+\ldots+A_{0}+\ldots+A_{s_{2}} x^{s_{2}}\right) R(x)^{-1}
$$

with $R(x) \in G L_{n}\left(\mathbf{C}\left[x, x^{-1}\right]\right), s_{1}+s_{2}=s$ is degree of the quasipolynomial matrix $A(x) R(x)$.

If (10) holds, then it is obvious that there exist matrices $N_{1}, N_{2}, \ldots, N_{s} \in M_{n}(\mathbf{C})$ such that

$$
A(x)=\left(E x^{-s_{1}}-N_{1} x^{-s_{1}+1}-\ldots-N_{s} x^{s_{2}}\right) x^{-s_{2}} x^{s_{2}} R_{1}(x),
$$

where the matrix $R_{1}(x)=A_{-s_{1}} R(x)^{-1} \in G L_{n}\left(\mathbf{C}\left[x, x^{-1}\right]\right)$.

Since $E x^{ \pm s} \in G L_{n}\left(\mathbf{C}\left[x, x^{-1}\right]\right)$ we have

$$
A(x)=\left(E x^{-s}-N_{1} x^{-s+1}-\ldots-N_{s-1} x^{-1}-N_{s}\right) R_{2}(x),
$$

where $R_{2}(x)=x^{s_{2}} R_{1}(x) \in G L_{n}\left(\mathbf{C}\left[x, x^{-1}\right]\right)$.

Left-multiplying the last equation by the matrix $P(x)$ from relation (1) and considering that $P(x) A(x)=S_{A}(x) Q(x)^{-1}$, we obtain

$$
P(x)\left(E x^{-s}-N_{1} x^{-s+1}-\ldots-N_{s-1} x^{-1}-N_{s}\right)=S_{A}(x) Q(x)^{-1} R_{2}(x)^{-1}
$$

or

$$
\left\|P(x) x^{-s},-P(x) x^{-s+1}, \ldots,-P(x) x^{-1},-P(x)\right\|\left\|\begin{array}{c}
E \\
N_{1} \\
\vdots \\
N_{s}
\end{array}\right\|=S_{A}(x) Q_{1}(x),
$$

where $Q_{1}(x)=Q(x)^{-1} R_{2}(x)^{-1} \in G L_{n}\left(\mathbf{C}\left[x, x^{-1}\right]\right)$.

Taking a certain number of derivatives (which depends on the multiplicity of a roots of the elements of diagonal matrix $S_{A}(x)$ ) and account the definition 5 , we obtain

$$
M_{P(x) x^{-s}}\left(S_{A}\right)-M_{P(x)\left\|E x^{-s+1}, \ldots, E x^{-1}, E\right\|}\left(S_{A}\right)\left\|\begin{array}{c}
N_{1} \\
\vdots \\
N_{s}
\end{array}\right\|=0 .
$$

This means that the linear inhomogeneous matrix equation

$$
M_{P(x)\left\|E x^{-s+1}, \ldots, E x^{-1}, E\right\|}\left(S_{A}\right)\left\|\begin{array}{c}
X_{1} \\
\vdots \\
X_{s}
\end{array}\right\|=M_{P(x) x^{-s}}\left(S_{A}\right)
$$

with unknown $n \times n$ matrices $X_{1}, X_{2}, \ldots, X_{s}$ has a solution.

The solution $\left\|\begin{array}{c}N_{1} \\ \vdots \\ N_{s}\end{array}\right\|$ is nonzero $\left(\operatorname{det} N_{s} \neq 0\right)$ and it is determined simultaneously $S_{A}(x)$ and $R(x)$.

Therefore, condition (9) is satisfied.

Sufficiency. By (1) for a matrix $A(x)$ we obtain 


$$
A(x)=P(x)^{-1} S_{A}(x) Q(x)^{-1}
$$

where $P(x), Q(x) \in G L_{n}\left(\mathbf{C}\left[x, x^{-1}\right]\right)$. By condition (10) it follows that matrix $P(x)^{-1} S_{A}(x)$ is right regularized, i.e. there exists matrix $Z(x) \in G L_{n}\left(\mathbf{C}\left[x, x^{-1}\right]\right)$ that $P(x)^{-1} S_{A}(x) Z(x)=B(x)$ is regular quasipolynomial matrix of degree $s$.

By (11) it follows that

$$
A(x) R(x)=B(x)
$$

where $R(x)=Q(x) Z(x) \in G L_{n}\left(\mathbf{C}\left[x, x^{-1}\right]\right)$.

The theorem is proved.

The following theorem establishes the uniqueness of the regularization of the Laurent polynomial matrix.

Theorem 5. The Laurent polynomial matrix $A(x)$ is right regularized uniquely.

Proof. Suppose that for a matrix $A(x)$ there exist matrices $R_{1}(x), R_{2}(x) \in G L_{n}\left(\mathbf{C}\left[x, x^{-1}\right]\right)$ such that

$$
A(x)=\left(A_{-s_{1}}^{(1)} x^{-s_{1}}+\ldots+A_{0}^{(1)}+\ldots+A_{s_{2}}^{(1)} x^{s_{2}}\right) R_{1}(x)^{-1}
$$

and

$$
A(x)=\left(A_{-s_{1}}^{(2)} x^{-s_{1}}+\ldots+A_{0}^{(2)}+\ldots+A_{s_{2}}^{(2)} x^{s_{2}}\right) R_{2}(x)^{-1} .
$$

If (12) and (13) are holds, then there exist matrices $N_{1}, N_{2}, \ldots, N_{s} \in M_{n}(\mathbf{C})$ and $H_{1}, H_{2}, \ldots, H_{s} \in$ $M_{n}(\mathbf{C})$ such that

$$
A(x)=\left(E x^{-s}-N_{1} x^{-s+1}-\ldots-N_{s}\right) \widetilde{R}_{1}(x)
$$

and

$$
A(x)=\left(E x^{-s}-H_{1} x^{-s+1}-\ldots-H_{s}\right) \widetilde{R}_{2}(x),
$$

where the matrices $\widetilde{R}_{i}(x)=x^{s_{2}} A_{-s_{1}}^{(i)} R_{i}(x)^{-1} \in G L_{n}\left(\mathbf{C}\left[x, x^{-1}\right]\right), i=1,2$.

Using the reasoning of Theorem 4 , we obtain linear inhomogeneous matrix equations

$$
M_{P(x)\left\|E x^{-s+1}, \ldots, E x^{-1}, E\right\|}\left(S_{A}\right)\left\|\begin{array}{c}
N_{1} \\
\vdots \\
N_{s}
\end{array}\right\|=M_{P(x) x^{-s}}\left(S_{A}\right)
$$

and

$$
M_{P(x)\left\|E x^{-s+1}, \ldots, E x^{-1}, E\right\|}\left(S_{A}\right)\left\|\begin{array}{c}
H_{1} \\
\vdots \\
H_{s}
\end{array}\right\|=M_{P(x) x^{-s}}\left(S_{A}\right)
$$

where matrices $N_{1}, N_{2}, \ldots, N_{s}$ and $H_{1}, H_{2}, \ldots, H_{s}$ are the solutions of equations (14) and (15), respectively.

Subtracting the left and right parts of equations (14) and (15), we obtain a homogeneous matrix equation with $\operatorname{det} M_{P(x)\left\|E x^{-s+1}, \ldots, E x^{-1}, E\right\|}\left(S_{A}\right) \neq 0$. This means that $N_{i}=H_{i}, i=1, \ldots, s$.

The theorem is proved.

Definition 7. Condition (9) is called the regularization condition of Laurent polynomial matrix.

By theorem 4, we obtain the method of finding the coefficients of the regular factor that stands out.

The matrix coefficients $N_{1}, N_{2}, \ldots, N_{s}$ of regular multiplier $B(x)=E x^{-s}-N_{1} x^{-s+1}-\ldots-N_{s}$ can be found by the formula:

$$
\left\|\begin{array}{c}
N_{1} \\
\vdots \\
N_{s}
\end{array}\right\|=\left[M_{P(x)\left\|E x^{-s+1}, \ldots, E x^{-1}, E\right\|}\left(S_{A}\right)\right]^{-1} M_{P(x) x^{-s}}\left(S_{A}\right) .
$$


Remark 2. Note that the regularization of Laurent polynomial matrix $A(x)$ is not obtained by regularization of the corresponding polynomial matrix $A(x) x^{l}$ where $l=-\underline{\operatorname{deg}} A(x)$, because the condition $n \mid \operatorname{deg} \operatorname{det} A(x) x^{l}$ where $n$ is order of matrix $A(x)$ is not always fulfilled.

Example 1. Let $A(x)=\left\|\begin{array}{cc}x^{-2} & x^{-2}-1 \\ x^{-1} & 0\end{array}\right\|$ be a Laurent polynomial matrix. This matrix is not regular. The Smith form $S_{A}(x)$ of $A(x)$ is equal to:

$$
S_{A}(x)=\left\|\begin{array}{cc}
1 & 0 \\
0 & x^{-1}-x^{-3}
\end{array}\right\|=\left\|\begin{array}{cc}
1 & 0 \\
-x^{-1} & 1
\end{array}\right\|\left\|\begin{array}{cc}
x^{-2} & x^{-2}-1\|\| \\
x^{-1} & 0
\end{array}\right\| \begin{array}{cc}
1 & 1-x^{-2} \\
-1 & x^{-2}
\end{array} \| .
$$

Let us check the regularization condition (9) of matrix $A(x)$. Degree of quasipolynomial $\operatorname{det} A(x)=x^{-1}-x^{-3}$ is equal 2, so a regular matrix can be a degree $s=1$. For system of roots $1,-1$ of elements of diagonal matrix $S_{A}(x)=\operatorname{diag}\left(1, x^{-1}-x^{-3}\right)$ we calculate the determinant of matrix of value $M_{P(x)}(1,-1)$ :

$$
\operatorname{det} M_{P(x)}(1,-1)=\left|\begin{array}{cc}
-1 & 1 \\
1 & 1
\end{array}\right|=-2 \neq 0,
$$

where $P(x)=\left\|\begin{array}{cc}1 & 0 \\ -x^{-1} & 1\end{array}\right\|$. Since it is nonzero, the regularization condition (9) is satisfied.

Therefore, by theorem 4 for matrix $A(x)$ there exists matrix $R(x) \in G L_{n}\left(\mathbf{C}\left[x, x^{-1}\right]\right)$ such that $A(x) R(x)$ is regular of degree $s=1$. We find the regular factor $B(x)=E x^{-1}-N_{1}$ by formula (16). Let us calculate values of matrix:

$$
M_{P(x) x^{-1}}(1,-1)=\left\|\begin{array}{cc}
-1 & 1 \\
-1 & -1
\end{array}\right\|,
$$

and we find the solution of the matrix equation (16): $N_{1}=\left\|\begin{array}{cc}0 & -1 \\ -1 & 0\end{array}\right\|$.

Hence $B(x)=\left\|\begin{array}{cc}x^{-1} & 1 \\ 1 & x^{-1}\end{array}\right\|$ is the regular matrix and $A(x) R(x)=B(x)$ with $R(x)=\left\|\begin{array}{cc}x & 1 \\ 0 & -1\end{array}\right\| \in$ $G L_{n}\left(\mathbf{C}\left[x, x^{-1}\right]\right)$.

\section{Factorization of Laurent polynomial matrices}

The theorem on regularization Laurent polynomial matrix can be successfully used to the problem of separation a regular factor with a predetermined of the Smith form from a nonsingular Laurent polynomial matrix. In this section we will formulate the necessary and sufficient conditions of the existence of the factorization

$$
A(x)=B(x) C(x)
$$

with $B(x)$ a regular quasipolynomial matrix and we indicate an efficient method for its actual construction. Let $\Phi(x)=\operatorname{diag}\left(\varphi_{1}(x), \varphi_{2}(x), \ldots, \varphi_{n}(x)\right)$ be a $d$-matrix and it is a divisor of the Smith form $S_{A}(x)(1)$ of Laurent polynomial matrix $A(x)$. Denote by

$$
V(\Phi)=\left\|\begin{array}{ccccc}
1 & 0 & \cdots & \cdots & 0 \\
\frac{\varphi_{2} k_{21}}{\left(\varphi_{2}, \varepsilon_{1}\right)} & 1 & \cdots & \cdots & 0 \\
\vdots & \vdots & \vdots & \ddots & \vdots \\
\frac{\varphi_{n} k_{n 1}}{\left(\varphi_{n}, \varepsilon_{1}\right)} & \frac{\varphi_{n} k_{n 2}}{\left(\varphi_{n}, \varepsilon_{2}\right)} & \cdots & \frac{\varphi_{n} k_{n n-1}}{\left(\varphi_{n}, \varepsilon_{n-1}\right)} & 1
\end{array}\right\|
$$


the matrix generated by the $d$-matrix $\Phi(x)$, where $\left(\varphi_{i}, \varepsilon_{j}\right)$ is a greatest common divisor of quasipolynomials $\varphi_{i}(x)$ and $\varepsilon_{j}(x), i, j=1, \ldots, n, i \geqslant j$,

$$
k_{i j}=\left\{\begin{array}{lll}
0, & \text { if } & \left(\varphi_{i}, \varepsilon_{j}\right)=\varphi_{j}, \\
k_{i j_{h_{i j}}} x^{-h_{i j}}+\ldots+k_{i j_{1}} x^{-1}+k_{i j 0}, & \text { if } & \left(\varphi_{i}, \varepsilon_{j}\right) \neq \varphi_{j},
\end{array}\right.
$$

$h_{i j}=\operatorname{deg} \frac{\left(\varphi_{i}, \varepsilon_{j}\right)}{\varphi_{j}}-1, i=2, \ldots, n, j=1, \ldots, n-1, i>j, k_{i j_{s}}$ are variables that join field $\mathbf{C}$, $s=0,1, \ldots, h_{i j}[12]$.

The following theorem establishes the necessary and sufficient conditions of a separation of a regular factor with a prescribed the Smith form from a Laurent polynomial matrix.

Theorem 6. Let $\Phi(x)$ be a $d$-matrix, $\operatorname{deg} \operatorname{det} \Phi(x)=n r$ and it is a divisor of the Smith form $S_{A}(x)(1)$ of Laurent polynomial matrix $A(x)$. The matrix $A(x)$ has a left regular divisor with the Smith form $\Phi(x)$ if and only if

$$
\operatorname{det} M_{V(\Phi) P(x)\left\|E x^{-r+1}, \ldots, E x^{-1}, E\right\|}(\Phi) \neq 0,
$$

where $P(x) \in G L_{n}\left(\mathbf{C}\left[x, x^{-1}\right]\right)$ in $(1), V(\Phi)$ has the form (18).

The proof of this theorem follows from theorem $2[16]$ and theorems 4, 5 of this article.

Theorem 7. Let $\Phi(x)$ be a $d$-matrix, $\operatorname{deg} \operatorname{det} \Phi(x)=n r$ and $A(x)$ has the Smith form $S_{A}(x)$. Suppose that

$$
S_{A}(x)=\Phi(x) \Psi(x) .
$$

The matrix $A(x)$ can be presented as $A(x)=B(x) C(x)$, where $B(x)$ and $\Phi(x), C(x)$ and $\Psi(x)$ are semiscalar equivalent matrices if and only if

$$
\operatorname{det} M_{P(x)\left\|E x^{-r+1}, \ldots, E x^{-1}, E\right\|}(\Phi) \neq 0,
$$

where $P(x) \in G L_{n}\left(\mathbf{C}\left[x, x^{-1}\right]\right)$ in $(1)$.

Proof. Since in (18) the conditions $\left(\varphi_{i}, \varepsilon_{j}\right)=\varphi_{j}$ hold for $i=2, \ldots, n, j=1, \ldots, n-1, i>j$, therefore the matrix $V(\Phi)=E$.

By theorem $3[16]$ we obtain the condition (21).

From theorem 6 follows the method of constructing of factorization (17) of Laurent polynomial matrix $A(x)$ with $B(x)=E x^{-r}-B_{1} x^{-r+1}-\ldots-B_{r}$ a regular quasipolynomial matrix of degree $r$ of Smith form

$$
\Phi(x)=\operatorname{diag}\left(\varphi_{1}(x), \varphi_{2}(x), \ldots, \varphi_{n}(x)\right)
$$

where matrix coefficients $B_{1}, B_{2}, \ldots, B_{r}$ can be found by the formula:

$$
\left\|\begin{array}{c}
B_{1} \\
\vdots \\
B_{r}
\end{array}\right\|=\left[M_{V(\Phi) P(x)\left\|E x^{-r+1}, \ldots, E x^{-1}, E\right\|}(\Phi)\right]^{-1} M_{V(\Phi) P(x) x^{-r}}(\Phi) .
$$

Condition (20) of theorem 6 provides the solvability of matrix equation (22).

Example 2. Let $A(x)=\left\|\begin{array}{cc}2 & -i\left(x^{-1}-x\right) \\ i\left(x-x^{-1}\right) & 2\end{array}\right\|$ be a matrix.

We calculate $\operatorname{det} A(x)=4-\left(x^{-1}-x\right)\left(x-x^{-1}\right)=2+x^{2}+x^{-2}$.

The Smith form $S_{A}(x)$ for $A(x)$ is equal to:

$$
S_{A}(x)=\left\|\begin{array}{cc}
1 & 0 \\
0 & 2+x^{2}+x^{-2}
\end{array}\right\|=\left\|\begin{array}{cc}
1 & 0 \\
\frac{-i}{2}\left(x-x^{-1}\right) & 1
\end{array}\right\|\left\|\begin{array}{cc}
2 & -i\left(x^{-1}-x\right) \\
i\left(x-x^{-1}\right) & 2
\end{array}\right\|\left\|\begin{array}{cc}
\frac{1}{2} & i\left(x^{-1}-x\right) \\
0 & 2
\end{array}\right\|,
$$

where $P(x)=\left\|\begin{array}{cc}1 & 0 \\ \frac{-i}{2}\left(x-x^{-1}\right) & 1\end{array}\right\| \in G L_{n}\left(\mathbf{C}\left[x, x^{-1}\right]\right)$. 
Since Smith form $S_{A}(x)$ of matrix $A(x)$ can be presented as:

$$
S_{A}(x)=\left\|\begin{array}{cc}
1 & 0 \\
0 & 2+x^{2}+x^{-2}
\end{array}\right\|=\left\|\begin{array}{cc}
1 & 0
\end{array} 0\right\| \begin{array}{cc}
1 & 0 \\
0 & 1+x^{-2}
\end{array}\|\| \begin{array}{cc}
0 \\
0 & 1+x^{2}
\end{array} \|
$$

and $\operatorname{deg} \operatorname{det} A(x)=4$, then we distinguish from the quasipolynomial matrix $A(x)$ a regular factor $B(x)$ of degree $r=1$ of Smith form $\Phi(x)=\operatorname{diag}\left(1,1+x^{-2}\right)$, $\operatorname{deg} \operatorname{det} \Phi(x)=2=n r$. By theorem 7 we have $V(\Phi)=E$.

For the system of roots $i,-i$ of elements of diagonal matrix $\Phi(x)=\operatorname{diag}\left(1,1+x^{-2}\right)$ let us calculate the matrices of values:

$$
M_{P(x)}(i,-i)=\left\|\begin{array}{cc}
1 & 1 \\
-1 & 1
\end{array}\right\|, \quad M_{P(x) x^{-1}}(i,-i)=\left\|\begin{array}{cc}
-i & -i \\
-i & i
\end{array}\right\|
$$

where $P(x) x^{-1}=\left\|\begin{array}{cc}x^{-1} & 0 \\ \frac{-i}{2}\left(1-x^{-2}\right) & x^{-1}\end{array}\right\|$.

We find the regular factor $B(x)=E x^{-1}-B_{1}$ by formula (22). Let us solve the matrix equation:

$$
B_{1}=\left\|\begin{array}{cc}
1 & 1 \\
-1 & 1
\end{array}\right\|^{-1}\left\|\begin{array}{cc}
-i & -i \\
-i & i
\end{array}\right\|=\left\|\begin{array}{cc}
0 & -i \\
-i & 0
\end{array}\right\| .
$$

Hence $B(x)=\left\|\begin{array}{cc}x^{-1} & i \\ i & x^{-1}\end{array}\right\|$ is a regular factor of degree $r=1$ of Smith form $\Phi(x)=\operatorname{diag}\left(1,1+x^{-2}\right)$.

Thus, $A(x)=\left\|\begin{array}{cc}x^{-1} & i \\ i & x^{-1}\end{array}\right\|\left\|\begin{array}{cc}x & -i \\ -i & x\end{array}\right\|$ is a required factorization of matrix $A(x)$.

\section{Conclusions}

In the context of this paper, the triangular form with invariant factors on the main diagonal, and the same as in Smith form, is obtained by means of semiscalar transformations for Laurent polynomial matrices. The theorem on regularization of Laurent polynomial matrices is proved. The latter result is important in solving the problem of isolating a regular multiplier with a predetermined Smith form from a nonsingular Laurent polynomial matrix. An efficient method for the actual construction of a factorization for Laurent polynomial matrix is indicated.

[1] Fornasini E., Valcher M.-E. $n$ D Polynomial Matrices with Applications to Multidimensional Signal Analysis. Multidimensional Systems and Signal Processing. 8 (4), 387-408 (1997).

[2] Kaczorek T. Polynomial and Rational Matrices: Applications in Dynamical System Theory. Commun. and Control Eng. Ser.; London (UK), Springer, (2007).

[3] Foster J. A., McWhirter J. G., Davies M. R., Chambers J. A. An algorithm for calculating the QR and singular value decompositions of polynomial matrices. AIEEE Trans. Signal Process. 58 (3), 1263-1274 (2010).

[4] Park I. Symbolic computation and signal processing. Journal of Symbolic Computation. 37, 209-226 (2004).

[5] McWhirter J. G., Baxter P. D., Cooper T., Redif S., Foster J. An EVD algo-rithm for Para-Hermitian polynomial matrices. IEEE Trans. Signal Process. 55 (6), 2158-2169 (2007).

[6] Kazimirskii P. S., Petrychkovych V. M. On the equivalence of polynomial matrices. Theoretical and Applied Problems of Algebra and Differential Equations. Lviv, 61-66 (1977).

[7] Petrychkovych V. M. On semiscalar equivalence and the Smith normal form of polynomial matrices. Journal of Mathematical Sciences. 66 (1), 2030-2033 (1993).

[8] Petrychkovych V. M. Generalized equivalence of pair of matrices. Linear Multi-linear Algebra. 48, 179-188 (2000). 
[9] Petrychkovych V. M. Standart form of pair of matrices with respect to generalized equivalence. Visnyk Lviv. Univ. 61, 153-160 (2003).

[10] Kuchma M. I. Symmetric equivalence of matrix polynomials and isolation of a common unital divisor in matrix polynomials. Ukrainian Mathematical Journal. 53 (2), 238-248 (2001).

[11] Dias da Silva J. A., Laffey T. A. On simultaneous similarity of matrices and related questions. Linear Algebra Appl. 291, 167-184 (1999).

[12] Kazimirskii P. S. Factorization of matrix polynomials. Lviv, Pidstryhach Institute for Applied Problems of Mechanics and Mathematics of the NAS of Ukraine, 2-nd edition, (2015), $282 \mathrm{p}$.

[13] Zelisko V. R., Kuchma M. I. Factorization of symmetric matrices over polynomial rings with involution. Journal of Mathematical Sciences. 96, 3017-3021 (1999).

[14] Zelisko V. R., Shchedryk V. P. Matrix of values on a system of roots of diagonal elements of matrix and its applications. Mat. Met. i Fiz.-Mekh. Polya. 48 (4), 20-29 (2005).

[15] Shchedryk V. P. Arithmetic of matrices over rings. Kyiv, Akademperiodyka (2021), 278 p.

[16] Kazimirskiy P. S., Shchedryk V. P. On solutions of matrix polynomials sides equations. Doklady AN SSSR. 304 (2), 271-274 (1989).

\title{
Трикутна форма поліноміальних матриць Лорана та їх факторизація
}

\author{
Кучма M. I. ${ }^{1}$, Гаталевич А. I. ${ }^{2}$ \\ ${ }^{1}$ Національний університет “Лъвівсъка політехніка", \\ вул. С. Бандери, 12, 79013, Львів, Україна \\ 2 Львівсъкий начіональний університет імені Івана Франка, \\ вул. Університетсвка, 1, 79000, Львів, Україна
}

\begin{abstract}
Досліджено питання напіскалярної еквівалентності поліноміальних матриць Лорана і встановлена відносно цієї еквівалентності трикутна форма таких матриць та їх скінченних наборів. Доведено теорему про регуляризацію для поліноміальних матриць Лорана. Ця теорема використовується у задачі факторизації таких матриць. Отримано критерій факторизації поліноміальних матриць Лорана із регулярним множником із наперед заданою нормальною формою Сміта.
\end{abstract}

Ключові слова: поліноміальна матрии, Лорана, напівскалярна еквівалентність, трикутна форма, нормальна форма Сміта, факторизація матриць. 